\title{
Semantics for Reciprocal Preferences
}

Fabián Castiblanco $^{a}$ and ${ }^{*}$ Camilo Franco $^{b}$ and $\mathbf{J}$. Tinguaro Rodríguez ${ }^{c}$ and Javier Montero ${ }^{c}$ ${ }^{a}$ Faculty of Economics, Administrative and Accounting Sciences, Gran Colombia University, Bogota 111711, fabianalberto. castiblanco@ugc.edu.co

${ }^{b}$ Faculty of Engineering, University of the Andes, Bogota 111711, c.franco310uniandes.edu.co

${ }^{c}$ Faculty of Mathematics, Complutense University, Madrid 28040

jtrodrig@mat.ucm.es, monty@mat.ucm.es

\begin{abstract}
Examining fuzzy preference relations together with the reciprocity condition, and a potential conceptual conflict arising between reciprocal fuzzy preferences and fuzzy preference structures, it is claimed that reciprocal preferences cannot be properly understood according to a weak nor a strict preference predicate. Therefore, as the elements of a standard preference structure, namely strict preference, weak preference, indifference and incomparability, cannot be properly distinguished from reciprocal fuzzy preferences, the concept of lack of preference emerges as a natural category, in fact allowing to understand reciprocal fuzzy preferences according to the standard fuzzy preference model. Furthermore, the basic notion of lack of preference is shown to group all the non-preference relations into a basic, unifying concept, providing an adequate decomposition of the meaning of any reciprocal fuzzy preference relation.
\end{abstract}

Keywords: Preference relations, fuzzy preference structures, reciprocity, additive reciprocity, preference semantics

\section{Introduction}

A decision problem can be defined according to a matrix of preferences, by providing a binary relation $R$ comparing every pair of available alternatives. This approach requires to address a number of difficulties, starting from the need of listing all alternatives to be considered, and most probably to have a quite detailed specification of each alternative. On one hand, it might be difficult to make explicit such a specification (many key human decision making processes, in particular those usually considered strategic, are quite often poorly defined). On the other hand, even in a decision making problem involving not many alternatives, the number of values $R(a, b)$ representing the degree to which each alternative $a$ is not worse (or strictly better) than each alternative $b$ might be overwhelming: with only 10 alternatives we have roughly 100 comparisons, and therefore 100 estimations. We can of course ask the decision maker to provide only some of those values, not all of them, and then design a procedure to address the problem with such partial information. But in order to ensure that such a procedure will produce interpretable results we usually need to a priori impose certain restrictions, so the provided information is consistent with a specific underlying mathematical model.

Considering the pair of evaluations we assign to any pair of alternatives $(a, b)$ in a binary or crisp framework, given by $R(a, b), R(b, a) \in\{0,1\}$, the meaning of each combination is clear, where the weak or global preference relation allows building up the whole structure of preferences. In this way, if $R(a, b)$ represents a weak preference of $a$ over $b$, then $R(a, b)=1, R(b, a)=0$ represents strict preference of $a$ over $b(a>b) ; R(a, b)=0, R(b, a)=1$ represents strict preference of $b$ over $a(b>a) ; R(a, b)=R(b, a)=1$ represents indifference between $a$ and $b$; and $R(a, b)=$ $R(b, a)=0$ represents incomparability between $a$ and $b$. A similar situation would appear if we had decided that $R(a, b)$ represents strict preference $(a>b)$. In this case, $R(a, b)=1, R(b, a)=0$ imply that $a \geq b$; $R(a, b)=0, R(b, a)=1$ imply that $b \geq a ; R(a, b)=$ $R(b, a)=0$ imply indifference; and $R(a, b)=R(b, a)=$ 1 imply incomparability.

Therefore, the meaning and semantical consistency of the values conforming the preference matrix should be subject to direct verification. Thus, within the binary framework, no matter the $\{0,1\}$ values we assign to $R(a, b)$ and $R(b, a)$, if consistent, they should allow obtaining a well-specified preference structure. In the 
same way, considering a fuzzy framework, the values assigned to the pairs $R(a, b), R(b, a) \in[0,1]$, should allow building the whole structure together with its corresponding degrees of strict preference $(P)$, weak preference $(R)$, indifference $(I)$ and incomparability $(J)$. Keep in mind that, in general [11], the desired structure of preferences cannot be obtained from any pair $R(a, b)$ and $R(b, a)$.

A key problem arises then, since some kind of reciprocity is commonly imposed to the fuzzy preference relation, in particular additive reciprocity, associated to a probabilistic setting, stating that for all $a, b \in \mathbb{A}, R(a, b)+R(b, a)=1$ should hold. As we will show in this paper (for an extended and more general study, see [2]), such a reciprocity might not be compatible with building the whole structure of preferences. In this sense, the semantic meaning of reciprocal valuations $R(a, b)$ and $R(b, a)$ is not explicitly clear, potentially producing inconsistencies if we assign either a weak or a strict preference meaning to both values.

In order to present the complete argument and outline the means to embed the corresponding meaning of fuzzy reciprocal preferences according to standard fuzzy preference structures, this paper is organized as follows: Section 2 discusses a seemingly failed relationship between the standard preference framework and reciprocal preferences. Taking a closer look, in Section 3, fuzzy reciprocal preferences are examined under the fuzzy standard model, showing that, in fact, they can be considered as equivalent to a special case of the standard model. Finally, some concluding remarks are provided in Section 4.

\section{On the meaning of reciprocal fuzzy preferences}

In this section, a first argument is presented, namely that reflexivity and reciprocity are incompatible properties, which cannot be simultaneously fulfilled by any relation $R$. Before doing so, let's recall some basics about the standard model for fuzzy preferences.

\subsection{Standard weak fuzzy preferences}

A fuzzy preference relation over any pair of alternatives $a, b \in \mathbb{A}$ is defined as a valued preference relation, such that $R(a, b): \mathbb{A}^{2} \rightarrow[0,1]$. Such a weak preference relation is assumed to be reflexive, i.e., $R(a, a)=1, \forall a \in \mathbb{A}$.

Following the standard fuzzy model [5, 11, 12], the decomposition of $R(a, b)$ allows the different relations of the standard preference structure $\langle P, I, J\rangle$ to be simultaneously verified up to certain degrees.

The standard fuzzy model [5] assumes Independence of Irrelevant Alternatives for the existence of continuous functions

$$
p, p^{-1}, i, j:[0,1]^{2} \rightarrow[0,1]
$$

such that the values of $P(a, b), P^{-1}(a, b), I(a, b)$ and $J(a, b)$ depend only on the pair $(a, b)$, through the weak preference functions $x=R(a, b)$ and $y=R(b, a)$. In this way, it holds that $P(a, b)=p(x, y), P^{-1}(a, b)=$ $p(y, x), I(a, b)=i(x, y)$ and $J(a, b)=j(x, y)$.

Besides, $p, p^{-1}, i, j$ are monotonic functions, such that $p(x, n(y)), p^{-1}(n(x), y), i(x, y)$ and $j(n(x), n(y))$ are non-decreasing over both arguments, where $n$ is a strict negation, and functions $i(x, y)$ and $j(x, y)$ are assumed to be symmetric (see again [5], but also [11] on a discussion about the different assumptions underlying this standard approach).

Therefore, the standard fuzzy preference structure is defined by the functions

$$
\begin{gathered}
P(a, b)=p(x, y), \\
I(a, b)=i(x, y), \\
J(a, b)=j(x, y) .
\end{gathered}
$$

In consequence, the classical preference properties (see e.g. [5]), can be formulated in fuzzy terms by means of De Morgan triples $(T, S, n)$, given by $t$-norms $T, t$-conorms $S$, and some strict negation $n$, verifying the condition

$$
n(S(x, y))=T(n(x), n(y)) .
$$

This leads to formulate such classical properties in fuzzy terms as follows:

$$
\begin{gathered}
x=S(p(x, y), i(x, y)), \\
S(x, y)=S\left(p(x, y), i(x, y), p^{-1}(x, y)\right), \\
n(x)=S\left(p^{-1}(x, y), j(x, y)\right), \\
S\left(p(x, y), p^{-1}(x, y), i(x, y), j(x, y)\right)=1 .
\end{gathered}
$$

It should be stressed that the standard model [5] presents a system of functional equations given by (4) and (6). Then, it can be shown (see [5]) that in order to fulfill such a system of equations (4), (6), the De Morgan triple $(T, S, n)$ has to be the Lukasiewicz De Morgan triple $\left(T^{L}, S^{L}, N\right)$. Up to a given automorphism $\varphi$ of the unit interval, the Lukasiewicz De Morgan triple is defined $\forall x, y \in[0,1]$, by $T^{L}(x, y)=\varphi^{-1}(\max \{\varphi(x)+\varphi(y)-1,0\}), S^{L}(x, y)=$ $\varphi^{-1}(\min \{\varphi(x)+\varphi(y), 1\})$ and the strong negation 
$N(x)=\varphi^{-1}(1-\varphi(x))$. Hence, the fuzzy properties (4)-(7) are stated in terms of the Lukasiewicz $t$ conorm, such that $S=S^{L}$

Besides, the functions $p, i, j$ of the standard fuzzy preference structure are usually defined (see again [5]) by means of continuous $t$-norms $T_{1}, T_{2}$, such that

$$
\begin{gathered}
p(x, y)=T_{1}(x, n(y)), \\
i(x, y)=T_{2}(x, y), \\
j(x, y)=T_{2}(n(x), n(y)) .
\end{gathered}
$$

Here it should be noted that $t$-norms may be chosen differently for strict preference $p(x, y)$ than for indifference $i(x, y)$ or incomparability $j(x, y)$.

Some particular and well known solutions for this standard fuzzy preference model can be recalled. On the one hand, it was shown [5] that the only solution for the fuzzy preference structure $(p, i, j)$, fulfilling the four properties (4)-(7), is given by $T_{1}=T^{M}$ and $T_{2}=T^{L}$, where the automorphism $\varphi$ is taken as the identity and $T^{M}(x, y)=\min \{x, y\}$, as in

$$
\begin{gathered}
p(x, y)=\min \{x,(1-y)\}, \\
i(x, y)=\max \{x+y-1,0\}, \\
j(x, y)=\max \{1-x-y, 0\} .
\end{gathered}
$$

The main feature of this solution is that indifference $i(x, y)$ and incomparability $j(x, y)$ are mutually exclusive, i.e., if $i(x, y)>0$, then $j(x, y)=0$ and vice versa.

On the other hand, another solution (besides the one given by (11)-(13)), which fulfills the system of equations (4), (6), but fails to verify property (5) (see [5], but also [12]), consists in defining strict preference $p$ as a strongly asymmetrical relation, by means of $T_{1}=T^{L}$, and $i, j$ through the minimum $T_{2}=T^{M}$ (hence allowing $i$ and $j$ to be simultaneously verified up to a specific degree). This solution is given by

$$
\begin{gathered}
p(x, y)=\max \{x-y, 0\}, \\
i(x, y)=\min \{x, y\}, \\
j(x, y)=\min \{1-x, 1-y\} .
\end{gathered}
$$

This second solution will be used in Section 3 to interpret reciprocal fuzzy preferences according to the standard fuzzy preference model.

\subsection{Incompatibility between standard and reciprocal fuzzy preferences}

In the following, the main argument on an inherent incompatibility between reciprocity and fuzzy preferences is outlined. First of all, given a (crisp or fuzzy) relation $R$ on $\mathbb{A}$, let us recall that $R$ is said to verify the reflexive property when for all $a \in \mathbb{A}$ it holds $R(a, a)=1$. Similarly, $R$ is said to verify the reciprocal property when for all $a, b \in \mathbb{A}$ it is $R(a, b)+R^{-1}(a, b)=1$, or equivalently, $R^{-1}(a, b)=1-R(a, b)$. Then notice that both properties collide in the diagonal of the preference matrix $R$, i.e., for all elements $a, b \in \mathbb{A}$ such that $a=b$, since it is well known that if $R$ is taken to be both reciprocal and reflexive, then $R$ should verify both that $R^{-1}(a, a)=1-R(a, a)$ and $R(a, a)=1$, which is impossible.

For this reason, a reciprocal relation cannot be in principle understood as a weak preference relation, which is assumed to be a reflexive relation. And vice versa, a weak preference relation cannot be reciprocal.

Nevertheless, it could be argued that reciprocity may only be imposed outside the diagonal of the relation $R$, that is $R^{-1}(a, b)=1-R(a, b)$ for all $a, b \in \mathbb{A}$ such that $a \neq b$. However, it is important to notice that this approach does not actually solve the problem. This is because if $a$ and $b$, although being different, are in practice very similar or even indistinguishable alternatives, so that indifference between $a$ and $b$ should hold, then in the framework of a weak relation it should hold that $R(a, b)=R^{-1}(a, b)=1$, but as mentioned above this is impossible under reciprocity. In other words, restricting reciprocity to hold only outside the diagonal of the relation still does not allow to understand reciprocal relations as weak preference relations. Rather, under reciprocity, both in the case of two practically indistinguishable alternatives $a \neq b$ and in the case of the diagonal pairs $a=b$, it has to be $R(a, b)=R^{-1}(a, b)=0.5$, in order to model such pairs of alternatives as being indifferent.

On the other hand, a reciprocal relation cannot be as well understood as a strict preference relation. Again, our main point will be focused on a sort of incompatibility of both notions over the diagonal of the relation or for very similar pairs. To this aim, let us recall a well established result of the standard fuzzy preference model [5].

Theorem 1. [5] If (4) and (6) are fulfilled by the function $p$, which defines strict preference as in (8), then $T^{L}(x, n(y)) \leq p(x, y) \leq T^{M}(x, n(y))$, where $T^{L}$ and $T^{M}$ are the Lukasiewicz and the minimum t-norm, respectively.

From this well known result, the following consequence is immediate.

Corollary 1. Let $R$ be a (reflexive) weak fuzzy preference relation, and let $P$ be a strict preference relation obtained from $R$ as a solution of the functional 
equations (4) and (6). Then, $P(a, a)=0$ for all $a \in \mathbb{A}$.

Proof. It is straightforward considering that, due to the reflexivity of $R$, it has to be $R(a, a)=1$, and then $P(a, a)=p\left(R(a, a), R^{-1}(a, a)\right)=p(1,1)=0$, since from the previous Theorem, it has to be $0=T^{L}(1,0) \leq$ $p(1,1) \leq T^{M}(1,0)=0$.

Thus, a reciprocal fuzzy preference relation cannot be a strict preference relation, due to the fact that strict preference cannot verify reciprocity over the diagonal. Hence, the preference predicate underlying a reciprocal relation has a different meaning than those of a weak or a strict fuzzy preference relation. In this sense, reciprocal relations might be considered to be outside the standard framework of fuzzy preference relations. In fact, a relevant question in our opinion is, what is the meaning of a reciprocal fuzzy relation? That is, how does the preference predicate underlying a reciprocal relation has to be understood? As we have seen, such a predicate of a reciprocal preference relation cannot be understood as at least as wanted as or as more wanted than.

In this same line, another reason leading us to consider reciprocal relations to be outside the standard fuzzy preference framework is that the $[0,1]$-valuation scale of reciprocal preferences receives a different meaning than the one of the standard model (which corresponds with the same $[0,1]$-valuation scale). In this sense, it can be shown that the standard model makes use of a univariate scale for measuring $R(a, b) \in[0,1]$, where 0 means lack of verification of the preference predicate " $a$ is at least as wanted as $b$ ", and 1 means a complete verification of the predicate (see [4], but also [6]). At the same time, the inverse predicate $R^{-1}(a, b)$ is separately measured on another univariate scale. Considered jointly, the pair $\left(R(a, b), R^{-1}(a, b)\right)$ can be simultaneously measured on a bi-variate scale given by the product of the two above mentioned univariate scales, $[0,1] \times[0,1]$.

On the contrary, reciprocal fuzzy preference relations make use of the same $[0,1]$ scale, but with a different meaning (also referred to as a univariate bipolar scale [4]. In this model, the values of 0 and 1 are respectively associated with preference of $b$ over $a$ and preference of $a$ over $b$. In this way, as opposed to the standard model where either $R(a, b)=0$ or $P(a, b)=0$ does not provide any information about the verification of the inverse predicate $R^{-1}(a, b)$ or $P^{-1}(a, b)$, the reciprocal model understands $R(a, b)=0$ as totally determining both predicates $R(a, b)$ and $R^{-1}(a, b)$.

From this standing point, it is not possible to explicitly relate reciprocal fuzzy preference to standard fuzzy preferences. But a closer look will allow us to examine the former as a special case of the latter.

\section{Reciprocity and fuzzy preference structures}

In this section, we recall some known results on reciprocal fuzzy preferences, and although it is acknowledged that reciprocal fuzzy preference relations do not belong to the standard frame, such a frame is used to investigate the meaning of reciprocal fuzzy preference relations. In this sense, it is shown that reciprocal preferences can be understood as equivalent to a special case of the standard model, being the notion of lack of preference the key to embed fuzzy reciprocal preferences within standard fuzzy preference structures.

\subsection{Some basics about reciprocal preference relations}

Reciprocal fuzzy preferences have been widely used in the literature (see e.g. [3, 7]), defined as a fuzzy relation $R(a, b)$ characterized by the mapping (its membership function) $R(a, b): \mathbb{A} \times \mathbb{A} \rightarrow[0,1]$, such that

$$
R(a, b)+R(b, a)=1, \quad \forall a, b \in \mathbb{A} .
$$

A reciprocal preference relation receives the following interpretation. If $R(a, b)=1$, then there is maximum degree of preference of $a$ over $b$, and if $R(a, b)=0$ (or equivalently $R(b, a)=1$ ), then maximum degree of preference, this time of $b$ over $a$, can be affirmed. And if $R(a, b)=0.5$, then maximum indifference exists between $a$ and $b$.

Therefore, the interval $(0.5,1)$ provides a transition between indifference and preference of $a$ over $b$, while the interval $(0,0.5)$ provides a transition between preference of $b$ over $a$ and indifference.

A common transformation that can be found in the literature (see e.g. $[3,1,8]$ ), provides a correspondence between fuzzy (weak) preference relations and reciprocal fuzzy relations. Such a transformation is given by

$$
G(a, b)=\frac{1+R(a, b)-R^{-1}(a, b)}{2}
$$

where it holds that $G^{-1}(a, b)=1-G(a, b)$, or equivalently, that $G(a, b)+G^{-1}(a, b)=1$.

Here it can be noted that some authors usually impose that the weak fuzzy preference relation $R$ has to be strongly complete, such that $R(a, b)+R(b, a) \geq 1$, 
in order to allow the previous correspondence to be applied over $R$.

So, for example, when $R(a, b)=1$ and $R^{-1}(a, b)=0$, it is obtained that $G(a, b)=1$, expressing preference of $a$ over $b$, and in case $R(a, b)=0$ and $R^{-1}(a, b)=1$, then $G(a, b)=0$, expressing inverse preference of $b$ over $a$. And in case both $R(a, b)=1$ and $R^{-1}(a, b)=1$, then $G(a, b)=0.5$, expressing maximum indifference.

In case the previous correspondence is applied over not (strongly) complete relations $R$, notice that when $R(a, b)=0$ and $R^{-1}(a, b)=0$, then it is also obtained $G(a, b)=0.5$, in principle expressing indifference. However, in the context of weak preference relations, the situation expressed by the pair $R(a, b)=0$ and $R^{-1}(a, b)=0$ corresponds with incomparability, as described above. This might explain why some authors restrict the previous correspondence to (strongly) complete preference relations.

Nevertheless, we think it is possible to apply the previous correspondence also to non-complete weak preference relations, if the (middle) value 0.5 of reciprocal relations is understood in a perhaps more general perspective, expressing lack of preference. Before going into this, it should be noted that, given any weak fuzzy preference relation, the previous correspondence of Eq. (18) provides a unique reciprocal relation, but not vice versa, as from a given $G$ it is not possible to uniquely determine both $R$ and $R^{-1}$. In order to obtain both $R$ and $R^{-1}$, given $G$, the following transformation can be used [8],

$$
R(a, b)=\min (2 G(a, b), 1) .
$$

Furthermore (see again [8]), both Eqs. (18) and (19) are inverse of each other, establishing a one-toone correspondence between strongly complete weak fuzzy relations and reciprocal fuzzy relations.

\subsection{Exploring the meaning of reciprocal preferences from standard preference structures}

Now, exploring the meaning of reciprocal fuzzy preference relations by means of the standard model, we can refer to a restricted preference structure, more in line with the classical preference structure $R=\langle P, I\rangle$ in a fuzzy setting. Therefore, Eq. (6) does not apply, since incomparability $J$ is not considered. Then, a solution can be found that will not verify Eq. (4), but will verify the fuzzy translation of the classical completeness property, namely, $S^{L}\left(p(x, y), p^{-1}(x, y), i(x, y)\right)=1$.

As a remark, it is observed that under this approach, the weak relation $R$ cannot be recovered from $P$ and $I$ (as it would be obtained if Eq. (4) is fulfilled), but also, that the resulting structure $\langle P, I\rangle$ leads to a loss of information with respect to the weak preference relation from which the structure is obtained.

Let us assume that we depart from a strongly complete weak preference $R$, such that $R(a, b)+R^{-1}(a, b) \geq 1$, and recall that $x=R(a, b)$ and $y=R(b, a)$. Then, this particular solution is given by the functions

$$
\begin{gathered}
p(x, y)=\max \{x-y, 0\}, \\
l(x, y)=1-|x-y|,
\end{gathered}
$$

where the notation $I(a, b)=l(x, y)$ for indifference is used instead of $i(x, y)$ for reasons that will become clear in a while.

Let us remark that this solution for the classical structure $R=\langle P, I\rangle$ offers a specific way of understanding how strict preference and indifference arise, or in other words, how they are obtained from the (reflexive) weak preference relation $R$. Actually, this solution is related to the second particular solution for the standard model presented in Section 2 (14)-(16), the main feature of which being that $P$ and $P^{-1}$ are mutually exclusive.

It is important to notice that this solution (20)-(21) crucially depends on the value of the difference $R(a, b)-R^{-1}(a, b)$. That is, the value of this difference determines both the strict preference $P$ and indifference $I$. For instance, if $(x, y)=(0.5,0.5)$, then it is $P(a, b)=p(x, y)=\max \{0.5-0.5,0\}=0$ and $I(a, b)=l(x, y)=1-|0.5-0.5|=1$. Instead, if alternatives $a, b$ are evaluated through a different pair $(x, y)$, but with the same difference $x-y$ as before, e.g., $(x, y)=(1,1)$, then it will be associated to the same preference description, i.e., $p(x, y)=0$ and $l(x, y)=1$. In other words, both $p(x, y)$ and $l(x, y)$ are invariant in the direction of the vector $(1,1)$.

Moreover, notice that, as a consequence of such an invariance, it is possible to obtain the values for $p(x, y)$ and $l(x, y)$ from $R$ and $R^{-1}$, but not the other way around. That is, given the strict preference and indifference valuations, respectively $P(a, b)$ and $I(a, b)$, it is not possible to recover $R(a, b)$ and $R^{-1}(a, b)$. To some extent, it can be said that this solution reduces the dimension or the amount of the available information.

However, a one-to-one correspondence between this solution and the reciprocal preference framework can be easily established. Recall that, as previously described, given a weak preference $R$ it is possible to obtain a reciprocal relation $G$ through Eq. (18). Then, 
it can be easily shown that the same reciprocal relation $G$ is obtained through the expression

$$
G(a, b)=P(a, b)+\frac{I(a, b)}{2},
$$

where $P$ and $I$ are obtained by the previous solution (20)-(21). That is, it holds that

$G(a, b)=P(a, b)+\frac{I(a, b)}{2}=\frac{1+R(a, b)-R^{-1}(a, b)}{2}$.

Importantly, this is a one-to-one correspondence, as we can recover $P(a, b)$ and $I(a, b)$ from $G(a, b)$ through the expressions

$$
P(a, b)= \begin{cases}0 & \text { if } G(a, b) \leq 0.5 \\ 2 G(a, b)-1 & \text { if } G(a, b)>0.5\end{cases}
$$

and

$$
I(a, b)= \begin{cases}2 G(a, b) & \text { if } G(a, b) \leq 0.5 \\ 2(1-G(a, b)) & \text { if } G(a, b)>0.5\end{cases}
$$

Moreover, the values for $P$ and $I$ obtained after (20)-(21) coherently reproduce the semantics of the reciprocal preference relation. For instance, when $P(a, b)=0$ and $I(a, b)=1$, it is obtained the value $G(a, b)=0.5$, which as discussed, expresses a maximum verification of indifference. Similarly, when $P(a, b)=1$ and $I(a, b)=0$, it is obtained $G(a, b)=1$, expressing maximum preference of $a$ over $b$. And the preference values $P^{-1}(a, b)=1$ and $I(a, b)=0$ lead to $G(a, b)=0$, expressing maximum preference of $b$ over $a$.

In summary, from a weak fuzzy preference relation $R$, a particular solution for the classical structure $\langle P, I\rangle$ is obtained by expressions (20)-(21). As shown, this structure is in a one-to-one correspondence with the reciprocal relation that would be obtained from $R$ through Eq. (18). And most importantly, the obtained values for $P$ and $I$ verify the semantics assigned in the literature to reciprocal fuzzy preferences obtained through the transformation (18). In other words, the particular solution just presented provides a $\langle P, I\rangle$ preference structure that can be considered as isomorphical to the framework of reciprocal fuzzy preference relations.

Nonetheless, it can be argued that the previous structure is not actually a solution of the standard fuzzy preference model, since, as it has been discussed, it is rather obtained in a relaxed setting and it does provide a somehow degenerate structure. However, let us now show that this particular solution can also be obtained through a proper solution of the standard fuzzy preference model in which indifference and incomparability collapse into a less informative situation of lack of preference.

Then, this proper solution of the standard model is given by Eqs. (14)-(16) of the second solution presented in Section 2, as in

$$
\begin{gathered}
P(a, b)=p(x, y)=\max \{x-y, 0\}, \\
I(a, b)=i(x, y)=\min \{x, y\}, \\
J(a, b)=j(x, y)=\min \{(1-x),(1-y)\} .
\end{gathered}
$$

Notice that $p(x, y)$ in Eq. (25) is the same as in Eq. (20) of the previous degenerate solution. Notice also that now it is not necessary to assume the strong completeness of the weak preference $R$. That is, the structure provided by this solution is applicable to any reflexive weak preference relation.

Furthermore, notice also that the function $l(x, y)$ defined in (21) coincides with the disjunction of indifference $i(x, y)$ and incomparability $j(x, y)$ defined via the functions (26) and (27), respectively. That is, it holds that

$$
l(x, y)=S^{L}(i(x, y), j(x, y))
$$

or equivalently, that

$$
\begin{aligned}
1-|x-y| & =S^{L}(\min \{x, y\}, \min \{(1-x),(1-y)\}) \\
& =\min \{x, y\}+\min \{(1-x),(1-y)\} .
\end{aligned}
$$

Thus, it follows that the $l(x, y)$ function of the degenerate solution can be considered to actually combine both the indifference $I$ and the incomparability $J$ of this proper solution. Or, in other words, $l(x, y)$ groups all the non-preference relations, i.e.,

$$
\begin{aligned}
l(x, y) & =N\left(S^{L}\left(p(x, y), p^{-1}(x, y)\right)\right) \\
& =1-\left(p(x, y)+p^{-1}(x, y)\right) .
\end{aligned}
$$

Therefore, it is more natural to interpret the situation modeled through this function $l(x, y)$ as lack of preference, rather than as indifference (see $[9,10]$ for an interesting discussion on the lack of information from a structural perspective). This is the reason why we used the notation $l(x, y)$ when presenting the previous degenerate solution (21). Following this idea, let us denote this situation of lack of preference by a relation $L$, such that

$$
L(a, b)=l(x, y)=i(x, y)+j(x, y) .
$$

Obviously, similarly to the above, there is a one-toone correspondence between the structure $\langle P, L\rangle$ and 
the reciprocal relation framework obtained through transformation (18), using the same mappings (23) and (24) as before, but replacing $I(a, b)$ by $L(a, b)$ in (24).

Another interesting feature of the lack of preference relation is that, due to the solution (25)-(27) verifying property (7), the resulting structure $\langle P, L\rangle$ provides a Ruspini partition over the values $G(a, b) \in[0,1]$ of a reciprocal fuzzy preference relation. In other words, it holds

$$
1=P(G(a, b))+L(G(a, b))+P^{-1}(G(a, b)),
$$

where $P(G(a, b)), L(G(a, b))$ and $P^{-1}(G(a, b))$ are obtained from $G(a, b)$ following expressions (23) and (24). In this way, the preference structure $\langle P, L\rangle$ provides an adequate decomposition of the meaning of any reciprocal fuzzy preference relation.

Summarizing, in our opinion these examples show that the framework of reciprocal fuzzy preferences can be regarded as isomorphic to a particular solution of the standard fuzzy preference model, in which indifference and incomparability collapse under the less informative situation of lack of strict preference. Particularly, this discussion suggests that the middle value $G(a, b)=0.5$ of a reciprocal fuzzy preference relation may actually express lack of preference rather than indifference, understanding that such a lack of preference situation also refers to incomparability besides indifference. Another point suggested by these examples is that the transformation (18) may also be applied to non complete weak fuzzy preference relations, since the semantics provided by the solution (25)-(27) applies to the whole unit square $[0,1]^{2}$ rather than only to the pairs $(x, y) \in[0,1]^{2}$ such that $x+y \geq 1$.

A final observation, already implicit in the above reasoning, is that the standard fuzzy preference model offers a more expressive and informative framework than that of reciprocal fuzzy preferences. In fact, these examples show that the framework of reciprocal preferences can be subsumed into the standard fuzzy preference framework. Particularly, the standard fuzzy preference model is able to express and distinguish between indifference and incomparability, while the other framework, that of reciprocal preferences, is only able to separate preference from lack of preference.

\section{Conclusions}

In this paper we have argued that reciprocal fuzzy preferences cannot be properly understood according to the weak preference predicate, i.e., that of being at least as wanted as, nor to the strict preference predicate, i.e., that of being more wanted than.

Moreover, it has been shown that reciprocal fuzzy preferences can be regarded as isomorphic to a particular solution of the standard fuzzy preference model, in which indifference and incomparability collapse under the less informative situation of lack of preference. In consequence, the standard fuzzy preference model allows expressing, as well as distinguishing, between indifference and incomparability. But once the reciprocal condition is imposed, the reduced model is only able to express and separate preference from lack of preference.

Therefore, careful attention should be given to preference models that make use of the reciprocity condition, checking the implications that the above semantical conflict (arising between reflexivity and reciprocity) may imbue over the model and its results.

\section{Acknowledgement}

This research has been partially supported by the Carolina Foundation (short postdoctoral research scholarship), the Government of Spain (grant PGC2018-096509-B-100), and Complutense University of Madrid (group research number 910149).

\section{References}

[1] B. D. Baets, H. D. Meyer, B. D. Schuymer, S. Jenei, Cyclic evaluation of transitivity of reciprocal relations, Social Choice and Welfare 26 (2006) 217-238.

[2] F. Castiblanco, C. Franco, J. Rodríguez, J. Montero, A characterization of reciprocal fuzzy preference structures and its compatibility with standard fuzzy preference structures, Fuzzy Sets and Systems (2021) In Press.

[3] F. Chiclana, E. Herrera-Viedma, S. Alonso, F. Herrera, Cardinal consistency of reciprocal preference relations: a characterization of multiplicative transitivity, IEEE Transactions on Fuzzy Systems 17 (2009) 14-23.

[4] D. Dubois, H. Prade, An introduction to bipolar representations of information and preference, International Journal of Intelligent Systems 23 (2008) 866-877.

[5] J. Fodor, M. Roubens, Fuzzy Preference Modelling and Multicriteria Decision Support, System Theory, Knowledge Engineering and Problem Solving, Kluwer Academic Publishers, Dordrecht, 1994.

[6] C. Franco, J. Rodríguez, J. Montero, Building the meaning of preference from logical paired 
structures, Knowledge-Based Systems 83 (2015) 32-41.

[7] E. Herrera-Viedma, F. Herrera, F. Chiclana, M. Luque, Some issues on consistency of fuzzy preference relations, European Journal of Operational Research 154 (2004) 98-109.

[8] D. Martinetti, S. Montes, S. Díaz, B. D. Baets, On the correspondence between reciprocal relations and strongly complete fuzzy relations, Fuzzy Sets and Systems 322 (2017) 19-34.

[9] J. Montero, H. Bustince, C. Franco, J. Rodríguez, D. Gómez, M. Pagola, J. Fernandez, E. Barrenechea, Paired structures in knowledge representation, Knowledge Based Systems 100 (2016) 50-58.

[10] J. Montero, D. Gómez, H. Bustince., On the relevance of some families of fuzzy sets, Fuzzy Sets and Systems 158 (2007) 2429-2442.

[11] J. Montero, J. Tejada, C. Cutello, A general model for deriving preference structures from data, European Journal of Operational Research 98 (1997) 98-110.

[12] B. V. D. Walle, B. D. Baets, E. Kerre, Characterizable fuzzy preference structures, Annals of Operational Research 80 (1998) 105-136. 